\title{
APPLICATION POSSIBILITY OF ELECTROHYDRODYNAMIC TECHNIQUE FOR CELLULOSE EXTRACTION FROM BAGASSE: OPTIMIZATION USING RESPONSE SURFACE METHODOLOGY
}

\author{
MARYAM BEYGY HARCHEGANI, ALI NASIRPOUR, SAFOURA AHMADZADEH, \\ JAVAD KERAMAT and NASSER HAMDAMI \\ Department of Food Science and Technology, College of Agriculture, \\ Isfahan University of Technology, Isfahan, Iran \\ \orresponding authors: A. Nasirpour, ali.nasirpour@cc.iut.ac.ir \\ S.Ahmadzadeh,s.ahmadzadeh@ag.iut.ac.ir
}

Received December 52017

\begin{abstract}
This study describes a promising alternative extraction process called electrohydrodynamic technique (EHD), used to extract cellulose from bagasse. The effects of EHD treatment time and heating time on cellulose extraction were investigated. The extraction conditions were then optimized using response surface methodology. The highest cellulose extraction yield was obtained when both independent variables were at their highest level (EHD: $2 \mathrm{~h}$; heating: $3 \mathrm{~h}$ ). A larger hydrodynamic size of cellulose was achieved under the optimum conditions, confirming the amorphous structure of cellulose created under the effect of EHD. Fourier-transform infrared spectroscopy was used to investigate the distribution of hydrogen bond types. The content of inter- and intra-molecular hydrogen bonds was altered when EHD was used for cellulose extraction. EHD significantly affected the composite-like structure of bagasse, which subsequently led to a higher cellulose extraction yield, and transition of crystalline to amorphous structure due to the disruption of the hydrogen bond network.
\end{abstract}

Keywords: sugarcane bagasse, cellulose extraction, electrohydrodynamic technique, response surface methodology

\section{INTRODUCTION}

In recent years, agro-industrial residues have been extensively investigated to accomplish more efficient applications. A reduction of the environmental impact, along with obtaining high profit, can be achieved by an efficient utilization of such agricultural wastes. ${ }^{1-3}$ In lignocellulosic materials, different constituents, such as hemicelluloses, lignin and pectin, act as noncellulosic cementing materials to make a composite structure embedding the cellulose fiber. $^{3-5}$ The cellulose polymer is the main component of lignocellulosic fibers. Cellulose has potential applications within different industries, including cosmetics, food industry, construction, electronic materials and pharmaceutical applications, in which the fibers are utilized as thickener, stabilizer, fat replacer, composite, texturizing agent or carrier for bioactive ingredients etc. ${ }^{6,7}$ Sugarcane bagasse (SCB), which is the major by-product acquired from the sugar industry, is a complex material that consists of cellulose, hemicelluloses and lignin. As a renewable material, the bagasse solid waste has huge potential to produce highly refined cellulose, which can be converted to some end-products, such as cellulose derivatives. ${ }^{7,8,11}$ The extraction of cellulose from SCB and its application to produce cellulose derivatives have been reported in the literature. ${ }^{7-12}$ A number of studies have been performed on developing bagasse processing methods, including alkaline, acidic and enzymatic treatments. $^{13-15}$ The complexity of the composition of lignocellulosic materials causes chemical agents to hardly penetrate into their structure, and therefore a pretreatment is required to be done to break this structure and facilitate the chemical processes. The pretreatments result in the fractionation and opening of lignocellulose. ${ }^{10,13}$

Cellulose Chem. Technol., 53 (3-4), 251-262(2019) 
Several methods have been considered in the literature as prehydrolysis processes, including ammonia fiber explosion, steam explosion, ozone, dilute acids and biological pretreatments, which facilitate the chemical hydrolysis through the explosion of cellular tissue and, subsequently, the separation of the components. ${ }^{14,17,18}$

The increasing global energy demand, as well as the application of energy-consuming processes, has forced different industries, including the food industry, to evaluate alternative processes. The electrohydrodynamic technique (EHD), as a nonthermal process, takes place under ambient pressure and temperature conditions, which makes it a suitable alternative to the conventional processes. EHD consumes less energy than conventional techniques and has a simple design, with no movable parts. As a branch of fluid mechanics, EHD is related to the fluid movement affected by electrical forces. It was reported that the electric field and the fluid motions influence each other. A high electric field accelerates the generation of electric wind by gaseous ions; which subsequently affects the application of EHD. The effect of EHD on heat transfer has been reported by Asakawa (1976). ${ }^{19}$ Since then, several studies have been done on EHD applications in different areas, including biomedical engineering and food processing, especially for drying processes. ${ }^{20-22}$ However, it should be noted that the interaction of high electric field with biological systems and food components is still unclear and more investigations need to be done to uncover the mechanisms of electric wind creation. Therefore, the application of EHD for complex biological matrices has yet to be investigated.

According to the literature, there is no reported work on the evaluation of EHD as a treatment used for cellulose extraction from SCB. The present work was conducted to investigate the possibility of EHD application as an innovation in isolating natural cellulose fibers, with uniform width, from bagasse. The high efficiency of EHD can overcome the difficulties of current processes used for cellulose extraction. Consequently, the environmental effects and the cost of the solventbased extraction process can be lowered by the application of EHD. However, the intensity of the electric field probably affects the obtained results.

In order to isolate cellulose fibers, SCB was subjected to a three-stage chemical process, involving chlorite delignification, bleaching and alkaline extraction, followed by EHD treatment. Moreover, this alternative method of cellulose extraction was optimized using response surface methodology (RSM). The effect of EHD treatment time and heating time on the structure of carbohydrates, structural properties and particle size were investigated. The hydrogen bonding network of cellulose was studied using infrared spectroscopy; which could be useful to predict the final properties of cellulose. The isolated cellulose was also submitted to chemical composition analysis.

\section{EXPERIMENTAL \\ Materials}

SCB was purchased from "Sugarcane \& ByProducts Development Company", Iran. It was cleaned, air dried and cut into small pieces. Afterwards, the sample was dried in an oven at $60{ }^{\circ} \mathrm{C}$. After grinding the cut bagasse, the fraction that passed through 35 mesh sieves was used for cellulose extraction. All the chemicals were purchased from Sigma Aldrich and were used as received.

\section{EHD set-up}

The electrohydrodynamic system was essentially equipped with a high-voltage power supply, and a needle-plane electrode system, in which a set of 45 steel needles with a diameter of $0.4 \mathrm{~mm}$ were fixed on a Plexiglas plate, which was placed horizontally above a fixed grounded plate made from copper $(20 \times 15$ $\mathrm{cm})$. The spacing between the neighboring needles was of $2.6 \mathrm{~cm}$. The discharge gap between the needle electrode and the plate electrode was set at $5 \mathrm{~cm}$. This system was embedded into a small wooden framework. The high-voltage power supply provided the maximum voltage level of $50 \mathrm{kV}$ and the output electric current of $5 \mathrm{~mA}$. The needle electrode was charged by connecting to high-voltage DC, through which the corona wind was formed.

\section{Cellulose extraction}

SCB samples were first defatted using a Soxhlet apparatus with hexane for $6 \mathrm{~h}$, which was applied to both control and EHD treated specimens. The main steps to extract the cellulose microfibers from SCB are described below.

Delignification: the prepared raw material was added to $0.7 \mathrm{wt} \%$ sodium chlorite solution at $\mathrm{pH} 4$ and treated under a high voltage electric field for 1-2 h. Delignification was then performed by boiling the mixture for $0-3 \mathrm{~h}$. In the case of the control sample, delignification was performed through boiling SCB in a sodium chlorite solution for $5 \mathrm{~h}$ without using EHD.

Bleaching: the obtained material was then washed with distilled water to attain a colorless material, mixed with $5 \mathrm{wt} \%$ sodium sulfite and treated under 
EHD for 1-2 h. Subsequently, the whole mixture was boiled for $0-3 \mathrm{~h}$ and finally rinsed with distilled water to attain a neutral $\mathrm{pH}$.

During this step, hemicelluloses were partially removed, and also lignin residues were completely eliminated. Similar to the previous step, the control sample was boiled for $5 \mathrm{~h}$ without using EHD.

Alkaline hydrolysis: in order to hydrolyze hemicelluloses, the obtained fibers were then treated with $17.5(\mathrm{w} / \mathrm{v}) \%$ sodium hydroxide solution under electrohydrodynamic treatment for $1-2 \mathrm{~h}$, and then under boiling conditions for $0-3 \mathrm{~h}(5 \mathrm{~h}$ for the control sample). Finally, the insoluble residue was filtered, washed until the filtrate was neutral, and dried at $80{ }^{\circ} \mathrm{C}$ until it reached a constant weight. ${ }^{23,24}$ The optimum extraction conditions were determined by using RSM.

\section{Chemical composition}

The chemical composition of SCB and extracted cellulose fibers was analyzed based on AOAC standard methods.

\section{Structural carbohydrates and lignin}

The content of cellulose, hemicelluloses and lignin were determined based on the standard of National Renewable Energy Laboratory U.S. (NREL) Analytical Procedure (TP-510-42618). ${ }^{25,26}$ A highperformance liquid chromatograph (HPLC) (Varian, United States), equipped with a UV-Vis detector, was applied to specify the concentrations of D-xylose, Larabinose, D-glucose, mannose, and galactose. HPLC grade water was used as the mobile phase. The content of cellulose and hemicelluloses was then calculated from the corresponding monomeric sugars. The weight percent of acid insoluble lignin and acid soluble lignin was also calculated according to the equations reported by Sluiter et al. ${ }^{26}$

Size exclusion chromatography (SEC)
A Varian Prostar high-performance liquid chromatograph, equipped with a pump and an ultraviolet (UV) absorbance detector (to monitor the elution profiles of cellulose), was used for measurement. Separation was performed in a SynChropak GPC-500 250×4.6 mm column, with tetrahydrofuran (THF) as the mobile phase, at a flow rate of $1 \mathrm{~mL} / \mathrm{min}$ and ambient temperature. The data were collected at $524 \mathrm{~nm}$. The carbanilation of samples was carried out according to the method reported by Stol et al..$^{27}$

\section{FTIR spectroscopy}

The infrared spectra of cellulose were recorded with a Bruker FTIR spectrophotometer (model Tensor 27, United States). Potassium bromide pellets containing $2 \%$ of finely ground cellulose fibers were used for this method. All the infrared spectra were recorded in a total of 4 scans for each sample, at a resolution of $4 \mathrm{~cm}^{-1}$ at $4000-400 \mathrm{~cm}^{-1}$.

The deconvolution of the spectra was performed based on the Gaussian distribution function, using Origin 8.0 software. The correlation values of $\mathrm{R}^{2} \geq$ 0.99 were observed for deconvolution fitting. Deconvolution caused resolving the $\mathrm{H}$-bonded $\mathrm{OH}$ stretching band at around $3300 \mathrm{~cm}^{-1}$ into three bands.

\section{Experimental design optimization}

RSM was used to optimize the cellulose extraction conditions. The experimental design was obtained using Design Expert 7. Two independent variables (EHD treatment time ( $\mathrm{h}, \mathrm{X}_{1}$ ) and heating time (h, $\left.\mathrm{X}_{2}\right)$ ) were applied to a D-Optimal Design. The coding and factors levels are described in Table 1. As presented in the table, three levels $(-1,0,1)$ were used to optimize the conditions. The effect of unintentional variability was minimized by randomly doing the experiments. ${ }^{28}$

Table 1

D-optimal design arrangement

\begin{tabular}{ccccc}
\hline \multirow{2}{*}{ Experiment } & \multicolumn{2}{c}{ Coded variables } & \multicolumn{2}{c}{ Real variables } \\
\cline { 2 - 5 } & $\mathrm{X}_{1}$ & $\mathrm{X}_{2}$ & EHD time $(\mathrm{h})$ & Heating time $(\mathrm{h})$ \\
\hline 1 & -1 & 1 & 1 & 3 \\
2 & -1 & -1 & 1 & 0 \\
3 & 1 & 1 & 2 & 3 \\
4 & -1 & 1 & 1 & 3 \\
5 & -1 & -1 & 1 & 0 \\
6 & 1 & -1 & 2 & 0 \\
7 & 1 & 1 & 2 & 3 \\
8 & -1 & 0 & 1 & 1.5 \\
9 & 0 & 0 & 1.5 & 1.5 \\
10 & 0 & -1 & 1.5 & 0 \\
11 & 1 & 0 & 2 & 1.5 \\
12 & 0 & 1 & 1.5 & 3 \\
\hline
\end{tabular}




\section{MARYAM BEYGY HARCHEGANI et al.}

Table 2

Chemical composition of sugarcane bagasse and extracted cellulose

\begin{tabular}{lcc}
\hline Components & Sugarcane bagasse & Cellulose fibers \\
\hline Moisture (wt $\%)$ & $3.18 \pm 0.07$ & $6.36 \pm 0.24$ \\
Protein (wt $\%)$ & $1.24 \pm 0.04$ & 0 \\
Ash (wt $\%)$ & $5.7 \pm 0.14$ & $0.46 \pm 0.02$ \\
Fat (wt $\%)$ & $1.0 \pm 0.15$ & 0 \\
Total carbohydrate (wt $)$ & $88.86 \pm 0.28$ & $93.35 \pm 0.22$ \\
\hline
\end{tabular}

Table 3

Chemical constituents of untreated and treated fibers based on EHD and heating time

\begin{tabular}{cccccc}
\hline \multirow{2}{*}{ Experiment } & \multicolumn{2}{c}{ Real variables } & \multicolumn{3}{c}{ Chemical constituents } \\
\cline { 2 - 6 } & EHD time (h) & Heating time (h) & Cellulose (\%) & Hemicelluloses (\%) & Lignin (\%) \\
\hline 1 & 1 & 3 & 68.1 & 18.7 & 5.4 \\
2 & 1 & 0 & 50.7 & 27.9 & 11.8 \\
3 & 2 & 3 & 77.8 & 13.8 & 3.6 \\
4 & 1 & 3 & 67.4 & 19.7 & 5.2 \\
5 & 1 & 0 & 47.6 & 27.8 & 12.7 \\
6 & 2 & 0 & 48.2 & 24.9 & 14.8 \\
7 & 2 & 3 & 78.3 & 15.6 & 3.0 \\
8 & 1 & 1.5 & 58.9 & 22.7 & 6.8 \\
9 & 1.5 & 1.5 & 69.7 & 21.0 & 3.8 \\
10 & 1.5 & 0 & 50.3 & 25.7 & 13.3 \\
11 & 2 & 1.5 & 76.1 & 18.1 & 4.3 \\
12 & 1.5 & 3 & 73.2 & 20.2 & 2.7 \\
SCB & & & 23.2 & 29.2 & 22.1 \\
Control sample & & & 60.8 & 23.8 & 7.3 \\
Microcrystalline cellulose (as reference) & 87.0 & 11.0 & 1.5 \\
\hline
\end{tabular}

\section{Statistical analysis}

Multiple linear regression was used to determine the regression coefficients for the linear and quadratic terms, as well as their interaction. The p-value was used to evaluate the statistical significance of the regression coefficient. The model was validated using the analysis of variance (ANOVA). The second order model of the response was then determined using the regression coefficients. The following equation indicates the model expressed with codified variables: $Y=\beta_{0}+\beta_{1} X_{1}+\beta_{2} X_{2}+\beta_{11} X_{1}^{2}+\beta_{22} X_{2}^{2}+\beta_{12} X_{1} X_{2}$ The variables are described as follows: $Y$ : the observed response; $\beta_{\mathrm{o}}$ : the constant for the equation; $\beta_{\mathrm{i}}$ : linear terms; $\beta_{\mathrm{ii}}$ : the quadratic terms for one variable; $\beta_{\mathrm{ij}}$ : the interaction terms.

Optimum extraction conditions were calculated according to the $2^{\text {nd }}$ order model. Design Expert 7 software was used to obtain the response surface plot as a function of independent variables.

\section{RESULTS AND DISCUSSION Chemical composition of fibers}

The chemical composition of SCB and extracted cellulose was analyzed and the results are presented in Table 2. It is clear that there are significant differences between the chemical composition of raw and treated fibers.

\section{Structural carbohydrates and lignin}

The chemical characterization of SCB was performed and the content of cellulose, hemicelluloses and lignin was determined as $23.2 \%, 29.2 \%$ and $22.1 \%$, respectively. The lignin content of the cellulose extracted under optimum conditions (EHD time: $2 \mathrm{~h}$, and heating time: $3 \mathrm{~h}$ ) was of $3.0 \%$, as given in Table 3 .

As presented in Table 3, increasing the extraction time results in higher extraction yield. Substantial differences were observed between the compositions of the fractions extracted under EHD treatment. Under the optimum extraction conditions, the yield of cellulose microfibrils from SCB was of $25.8 \%$, and the total recovered cellulose percent was of about $87 \%$. The loss of cellulose is probably caused by partial degradation of cellulose or washing the treated materials with distilled water until neutral during the isolation of cellulose. ${ }^{29,30}$ 
The content of cellulose and hemicelluloses in the extracted fibers was in the range from $47.6 \%$ to $78.3 \%$ and from $13.8 \%$ to $27.9 \%$, respectively, which, in comparison with the values determined for SCB fibers (23.2\% and $29.2 \%$, respectively), confirms an effective removal of non-cellulosic compounds, such as pectin, lignin and wax, through EHD and chemical treatments. The concentrations of xylose, as the main component of hemicelluloses, and glucose, as the main component of cellulose, were monitored under the effect of the treatment conditions, and considered as the content of hemicelluloses and cellulose, respectively. The efficiency of the hydrolysis process can be evaluated based on the concentration of hemicelluloses remaining after the process. The results indicated that the yield of xylose was influenced by both variables; therefore, the maximum extraction of xylose can be obtained by keeping a compromise between the heating time and the time of EHD treatment. It is well known that a composite-like structure, comprising non-cellulosic materials binding the fiber bundles, is present in the form of network. This structure is weakened under the effect of the electric field and alkali treatment because of the removal of hemicelluloses, which subsequently causes lignin to be removed from the network. The electroporation mechanism can be considered as an explanation for the mechanical rupture and the increase of mass permeability under the effect of EHD, which leads to an increase in the extraction yield, and also to a change of the cellulose characteristics. However, the special chemical agents used for the process of extraction also affect the properties of cellulose in different ways. The extraction of hemicelluloses can be significantly facilitated after delignification using chlorite, which results in high purity of the isolated cellulosic polymer. Through this chemical process, the lignin is removed without any noticeable hemicelluloses and cellulose degradation, and thereafter, the cellulose and hemicelluloses are separated by an alkaline process. ${ }^{31}$ Cellulose and hemicelluloses interact with each other presumably via hydrogen bonds. ${ }^{32}$ It is believed that hemicelluloses can crosslink the adjacent cellulose microfibrils or bind to the surface of microfibrils, forming a network embedded in a matrix containing hemicelluloses involved in different types of non-covalent crosslinks. The hemicelluloses chains may be crosslinked through hydrophobic interactions and hydrogen bonds. ${ }^{33}$ In addition, another chemical crosslink may be formed between the ferulic acid contained in the side chains of hemicelluloses, which results in a covalent network in which the complex of cellulose-hemicelluloses may be embedded. $^{34}$ Sodium sulfite used for the bleaching process absorbs the complex of ligninchlorine, which is formed through bagasse digestion with sodium chlorite, and is highly soluble in the sodium sulfite solution. Moreover, hemicelluloses can also be extracted into the sodium sulfite solution under boiling conditions. ${ }^{30}$ A higher degree of xylan chains branching means higher content of extracted arabinose, which indicates higher solubility of hemicelluloses due to the easy solubilization of arabinose, as a side chain in hemicelluloses, during the initial extraction process. The sequential alkali treatment causes partial cleavage or degradation of this side chain. $^{15}$ Cellulosic fractions contain small amounts of hemicelluloses, suggesting strong resistance of some parts of hemicelluloses against the alkaline treatment under used conditions. This phenomenon also implied the association of hemicelluloses to the pores, in addition to the cellulose surface through hydrogen bonds, which can cause hemicelluloses to remain in the network of fibrils during alkali treatment. ${ }^{1}$ The percentages of hemicelluloses and lignin decreased in the treated fibers from $29.2 \%$ to $13.8 \%$, and from $22.1 \%$ to $3.0 \%$, respectively. These observations confirm that the lignocellulosic structure was substantially broken down, the hemicellulosic fraction was partially hydrolyzed, and the lignin components were depolymerized under the chemical extraction process followed by EHD treatment. However, these components were not completely removed. Partial hydrolysis of hemicelluloses and the depolymerization of lignin give rise to water soluble compounds, such as sugars and phenolic compounds. Under EHD treatment, the carbohydrate fraction is rapidly hydrolyzed to monomeric sugars, resulting in greater yields of cellulose extraction. Moreover, autohydrolysis occurs at high temperature, at which the acetic acid formed from the hemicellulosic acetyl groups catalyzes the hydrolysis of glycosidic bonds in hemicelluloses and the ether bonds in lignin. ${ }^{35}$ In general, the results indicated that the yield of cellulose isolation was significantly influenced by EHD treatment, probably due to the rapid electrical breakdown. However, as mentioned in the previous section, the interaction of EHD with biological systems and food components is still 
unclear, and more investigations need to be done to uncover the mechanisms involved in the extraction process.

\section{Optimization of experiments}

The cellulose extraction conditions were optimized using RSM. Two input variables (heating time and EHD treatment time) were considered in the optimization selection in order to set desirability indices.

Design Expert 7 software was used to analyze the collected data. All the measured factors were evaluated using ANOVA and regression analysis to fit the model and estimate the significance of the coefficient terms. The results of the analysis of variance are presented in Table 4 . The p-values calculated for the responses were used to evaluate the regression coefficients related to independent variables. The multiple regression coefficients that were significant $(\mathrm{p} \leq 0.05)$ were used to develop the model. According to the ANOVA results, the quadratic regression model of the cellulose content was significant based on the low probability F-test value $(p=0.0002)$. The F-test values of the model related to the hemicelluloses and lignin contents were 0.0001 and 0.0002, respectively, implying the models were significant. However, the lack of fit of 10.61 and 13.76, respectively, corresponding to the cellulose and lignin content, indicated the significance of the lack of fits as well. Therefore, further analysis was required for the models, which was performed by considering the $\mathrm{R}^{2}$ value. The $\mathrm{R}^{2}$ of the models were calculated as $0.95,0.958$ and 0.948 for cellulose, hemicelluloses and lignin content, respectively.

It was concluded that the models can be used to predict the cellulose content of the fibers extracted from SCB within the experimental limits. The heating time indicated the highest value of the regression coefficient for the cellulose content; therefore, it can be figured out that the heating time had a greater effect on the cellulose extraction than the EHD treatment time, as shown in Table 5.

The results indicated that the best fit for the cellulose yield was obtained by the quadratic regression model. The estimated models for cellulose, hemicelluloses and lignin are demonstrated in terms of the coded values in Table 5; however, these equations are only validated within the tested range of the independent variables (heating time: $0-3 \mathrm{~h}$; and EHD treatment time: $1-2 \mathrm{~h}$ ).

Table 4

Analysis of variance of cellulose, hemicelluloses and lignin contents (for the quadratic model)

\begin{tabular}{lccccc}
\hline \multicolumn{5}{c}{ Cellulose } \\
\hline Source & Sum of squares & DF & Mean squares & F value & P value \\
\hline Model & 1525.86 & 5 & 305.37 & 26.70 & 0.0002 (Significant) \\
Residual & 80.07 & 1 & 11.44 & & \\
Lack-of-fit & 74.79 & 4 & 18.70 & 10.61 & 0.047 (Significant) \\
Pure error & 5.29 & 3 & 1.76 & & \\
Total & 1606.93 & 12 & & & \\
\hline \multicolumn{7}{c}{ Hemicelluloses } & & \\
\hline Model & 221.62 & 5 & 44.32 & 32.13 & 0.0001 (Significant) \\
Residual & 9.66 & 7 & 1.38 & & \\
Lack-of-fit & 6.90 & 4 & 1.73 & 1.88 & 0.3156 (In significant) \\
Pure error & 2.75 & 3 & 0.92 & & \\
Total & 231.28 & 12 & & & \\
\hline \multicolumn{7}{c}{} & \multicolumn{7}{c}{ Lignin } & & \\
\hline Model & 213.12 & 5 & 42.62 & 25.59 & 0.0002 (Significant) \\
Residual & 11.66 & 7 & 1.67 & & \multirow{2}{*}{0.0285 (Significant) } \\
Lack-of-fit & 11.66 & 4 & 2.76 & 13.76 & \\
Pure error & 0.6 & 3 & 0.20 & & \\
Total & 224.77 & 12 & & & \\
\hline
\end{tabular}


Table 5

Regression coefficients and p-values of approximate polynomials for cellulose, hemicelluloses and lignin contents in experimental design

\begin{tabular}{|c|c|c|c|c|c|c|}
\hline \multirow{2}{*}{ Term } & \multicolumn{2}{|c|}{ Cellulose content $\left(\mathrm{Y}_{1}\right)$} & \multicolumn{2}{|c|}{ Hemicelluloses content $\left(\mathrm{Y}_{2}\right)$} & \multicolumn{2}{|c|}{ Lignin content $\left(\mathrm{Y}_{3}\right)$} \\
\hline & Coefficient & P-value & Coefficient & P-value & Coefficient & P-value \\
\hline Constant & 67.56 & - & 21.02 & - & 5.28 & - \\
\hline $\mathrm{X}_{1}$-EHD time & 3.8 & 0.0137 & -2.01 & 0.0016 & -0.12 & 0.7956 \\
\hline $\mathrm{X}_{2}$-Heating time & 11.91 & $<0.0001$ & -4.017 & $<0.0001$ & -4.86 & 0.0001 \\
\hline $\mathrm{X}_{1} \mathrm{X}_{2}$ & 2.12 & 0.1385 & -0.37 & 0.4297 & -0.82 & 0.1328 \\
\hline $\mathrm{X}_{1}{ }^{2}$ & -1.24 & 0.6026 & -1.14 & 0.1926 & -1.23 & 0.1990 \\
\hline $\mathrm{X}_{2}^{2}$ & -4.71 & 0.0024 & 1.93 & 0.0009 & 1.99 & 0.0013 \\
\hline $\begin{array}{l}\text { Quadratic polynominal model } \\
\text { R-square }\end{array}$ & \multicolumn{2}{|c|}{$\begin{array}{c}\mathrm{Y}_{1}=67.56+3.8 \mathrm{X}_{1}+11.91 \mathrm{X}_{2}-4.71 \mathrm{X}_{2}^{2} \\
0.952\end{array}$} & \multicolumn{2}{|c|}{$\begin{array}{c}\mathrm{Y}_{2}=21.02-2.01 \mathrm{X}_{1}-4.017 \mathrm{X}_{2}+1.93 \mathrm{X}_{2}^{2} \\
0.958\end{array}$} & \multicolumn{2}{|c|}{$\begin{array}{c}Y_{3}=5.28-4.86 X_{2}+1.99 X_{2}^{2} \\
0.948\end{array}$} \\
\hline
\end{tabular}
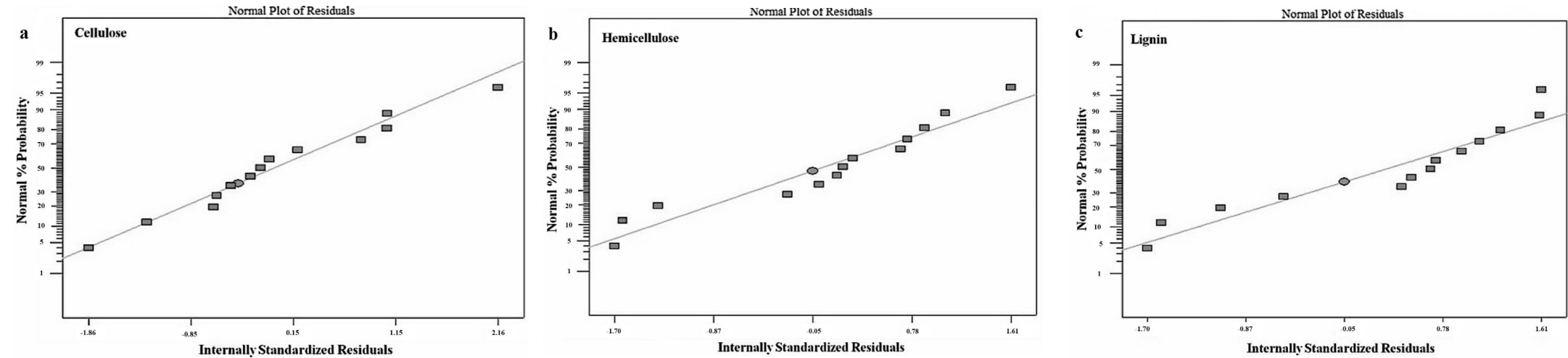

Figure 1: Normal probability plots of residuals 


\section{MARYAM BEYGY HARCHEGANI et al.}
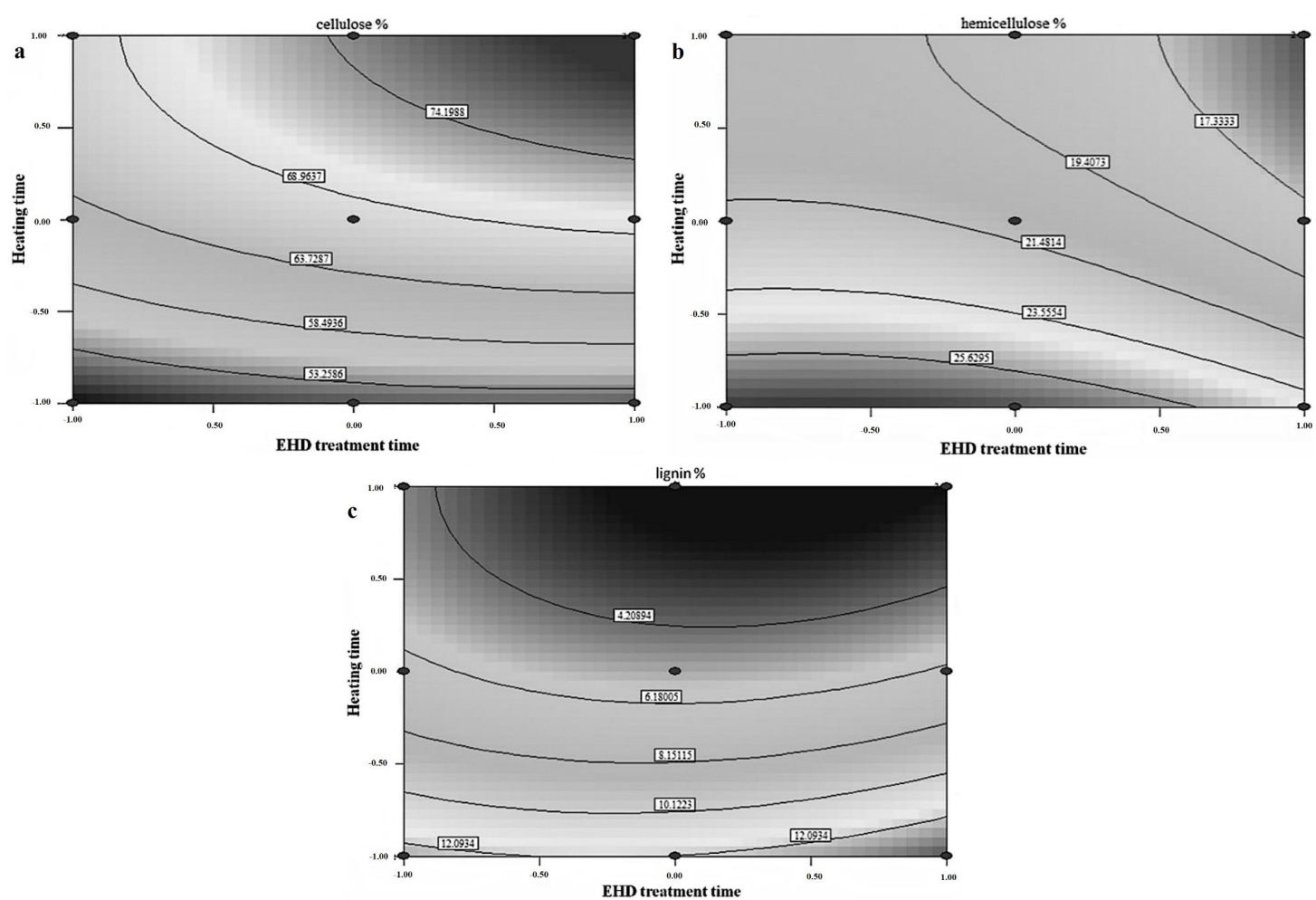

Figure 2: Contour plots of interactive effects of EHD treatment time and heating time on RSM

The residuals should be examined to evaluate the adequacy of the model. Residuals analysis was performed to confirm that the assumptions for the analysis of variance are met. Normal plots of residuals were used to examine the residuals. ${ }^{28,36}$ The normal plots of the residuals are shown in Figure 1. These plots are in a straight line, implying normal distribution of the errors.

According to the equations, the contour plots of the effects of heating time and EHD treatment time, as independent variables, on the cellulose, hemicelluloses and lignin content are shown in Figure 2. It can be clearly observed that the independent variables quadratically affect the response values. However, the most significant effect corresponded to the heating time. The cellulose content increased with increase the heating time.

The target values were the highest amount of cellulose and the lowest amounts of hemicelluloses and lignin obtained from the experimental results. As shown in Table 3, the highest cellulose content was obtained when the heating time and EHD treatment time were at their highest level within the ranges tested. Therefore, the optimum condition of the independent variables was determined as $2 \mathrm{~h}$ for EHD treatment time and $3 \mathrm{~h}$ for heating time.

\section{Cellulose chromatograms: ultraviolet absorbance}

The size exclusion chromatography (SEC) technique is a special type of chromatography, in which the separation occurs according to the hydrodynamic volume of the molecules, and no interaction happens between the stationary phase and the molecules. In this method, large molecules are eluted first, while small ones are eluted later, as the solid phase passes through the pores. The hydrodynamic radius is influenced by the conformation of the molecule, branching and the interaction between the solvent and the molecule. Generally, the hydrodynamic volume of molecules is the only factor influencing the separation by using SEC. ${ }^{37}$ In this study, the effect of cellulose extraction conditions on the molecular size of cellulose was evaluated by the SEC technique. The chromatograms obtained for the samples were overlaid to compare their quality (Fig. 3). A significant peak with retention time at 3.384 min was observed from the SCB chromatogram. 


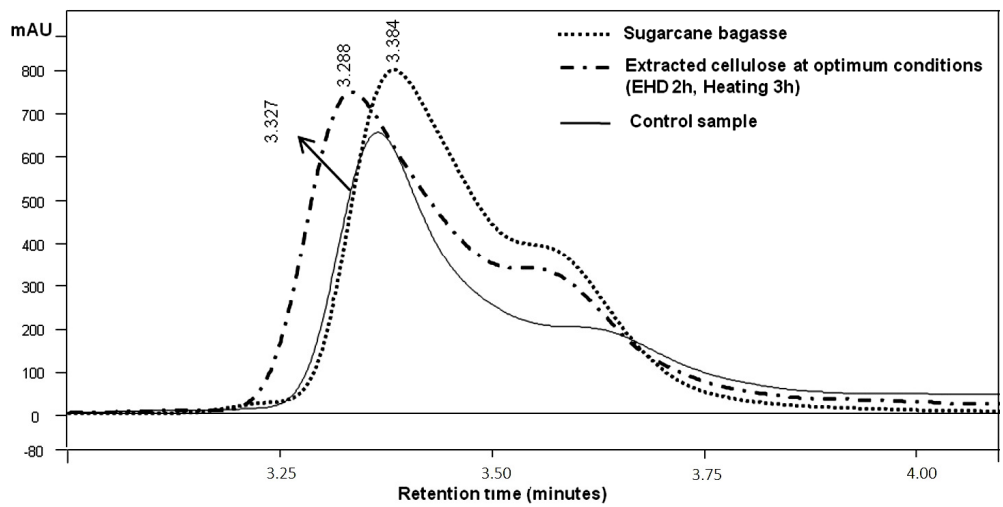

Figure 3: Chromatograms recorded at $524 \mathrm{~nm}$, using a UV detector, for SCB, control sample and cellulose extracted under the optimum conditions

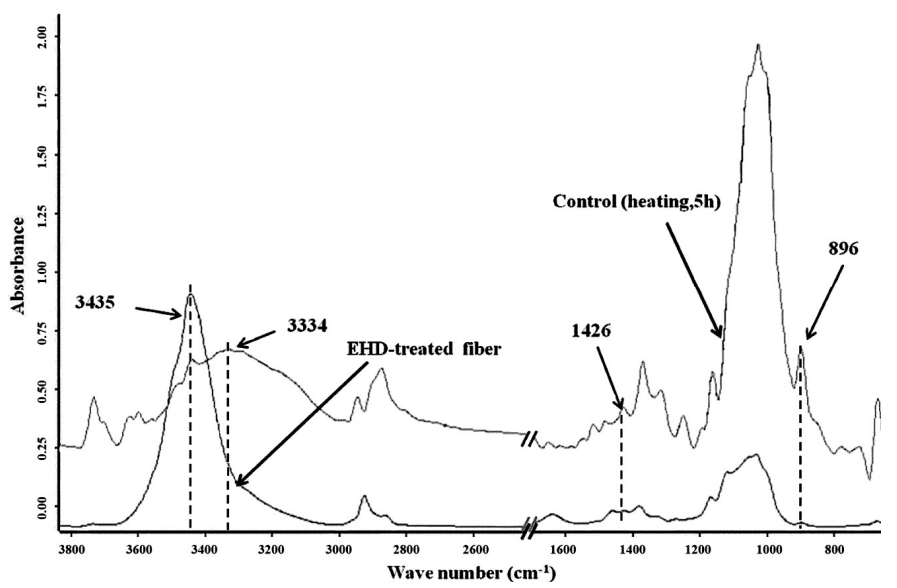

Figure 4: FTIR spectra of extracted cellulose (EHD-treated and control specimens)

Based on the size exclusion theory, it can be figured out that the samples having larger hydrodynamic radius present an upstream peak, while the molecules having smaller hydrodynamic radius exhibit a downstream peak. ${ }^{38}$ It was observed that a larger size of cellulose molecules is achieved under the optimum conditions of extraction, which could be due to the amorphous structure of cellulose created under the effect of EHD. The amorphous structure of cellulose contains a higher amount of hydrogen bonds, which cause the size of cellulose fibers to increase. This result was confirmed by FT-IR spectroscopy analysis (Fig. 4).

\section{Hydrogen bond analysis}

It was reported that a strong band at about $3340 \mathrm{~cm}^{-1}$, assigned to $\mathrm{OH}$ stretching, indicates a large number of hydroxyl groups, which confirms the formation of more hydrogen bonds. ${ }^{39}$ The obtained results indicated that EHD-treated samples showed higher absorbance at $3435 \mathrm{~cm}^{-1}$, compared to MCC and the control specimens, which confirms a higher proportion of hydrogen bonds (Figs. 4, 5). Moreover, two peaks at 1426 $\mathrm{cm}^{-1}$ and $896 \mathrm{~cm}^{-1}$, which are related to the crystalline structure, almost disappeared in the FTIR spectra of EHD-treated samples, indicating their higher proportion of amorphous region, compared to the control sample (Fig. 4).

The hydrogen bonds in cellulose are altered under the effect of some chemical and physical transformations. The cellulose polymer chains contain a large number of hydroxyl groups, which contribute to the formation of intra- and intermolecular hydrogen bonds. It has been reported that the chemical, physical and mechanical properties of cellulose are strongly affected by the hydroxyl groups located in the positions of $\mathrm{C}_{2}, \mathrm{C}_{3}$ and $\mathrm{C}_{6}$. 
As shown in Figure 5, the $\mathrm{H}$-bonded $\mathrm{OH}$ stretching vibration related to microcrystalline cellulose (MCC), the control and EHD-treated samples are resolved into three bands, which can be attributed to the intra- and inter-molecular hydrogen bonds. It has been reported that, in the cellulose I structure, the infrared bands related to the two types of hydrogen bonds (intra-molecular: $\mathrm{O}(2) \mathrm{H} . . . \mathrm{O}(6), \mathrm{O}(3) \mathrm{H} . . \mathrm{O}(5)$; and inter-molecular: $\mathrm{O}(6) \mathrm{H} . . \mathrm{O}(3))$ appear at $3455-3410 \mathrm{~cm}^{-1}, 3375$ $3340 \mathrm{~cm}^{-1}$ and $3310-3230 \mathrm{~cm}^{-1}$, respectively. ${ }^{40}$ In the cellulose II structure, these bands appear at about $3486 \mathrm{~cm}^{-1}, 3374 \mathrm{~cm}^{-1}$ and $3309 \mathrm{~cm}^{-1}$, respectively, ${ }^{41}$ which is in accordance with the results obtained in the present study. For the cellulose extracted under EHD treatment, $\mathrm{H}$ bonded $\mathrm{OH}$ stretching shifted to a higher wavenumber $\left(3435 \mathrm{~cm}^{-1}\right)$ (Fig. 4), which is related to the change of the proportion of hydrogen bond types. The crystallinity of MCC and EHD-treated samples was measured to be about $86 \%$ and $12.96 \%$, respectively. ${ }^{42}$ It was proposed by Kondo and Sawatari ${ }^{43}$ that higher crystallinity can be created by a higher contribution of the $\mathrm{C}_{6}$ hydroxyl group in the formation of intermolecular hydrogen bonds, as can be observed in Figure 5 (MCC). Moreover, they reported that higher formation of intermolecular $\mathrm{H}$-bonds at the positions of $\mathrm{C}_{2}, \mathrm{C}_{3}$ and $\mathrm{C}_{6}$ can be considered as an effective factor to estimate the proportion of crystalline and amorphous structure. It was reported by Langan et $a l .{ }^{44}$ that in the cellulose II structure, $\mathrm{O}(6) \mathrm{H} . . \mathrm{O}(3)$ and $\mathrm{O}(6) \mathrm{H} . . . \mathrm{O}(5)$ (as inter-molecular H-bonds) are minor components, while the major intra-molecular hydrogen bond is $\mathrm{O}(3) \mathrm{H} . . . \mathrm{O}(5)$ along with a minor component of $\mathrm{O}(3) \mathrm{H} . . . \mathrm{O}(6)$. This proportion probably changes under the effect of EHD, which causes the $\mathrm{H}$ bonded $\mathrm{OH}$ stretching vibration to shift. As shown in Figure 5, a significant difference was observed between the MCC, control and EHDtreated samples. The results indicated that the content of inter-molecular hydrogen bonds significantly decreased by using EHD for the extraction process; confirming a high proportion of the amorphous structure in this specimen.
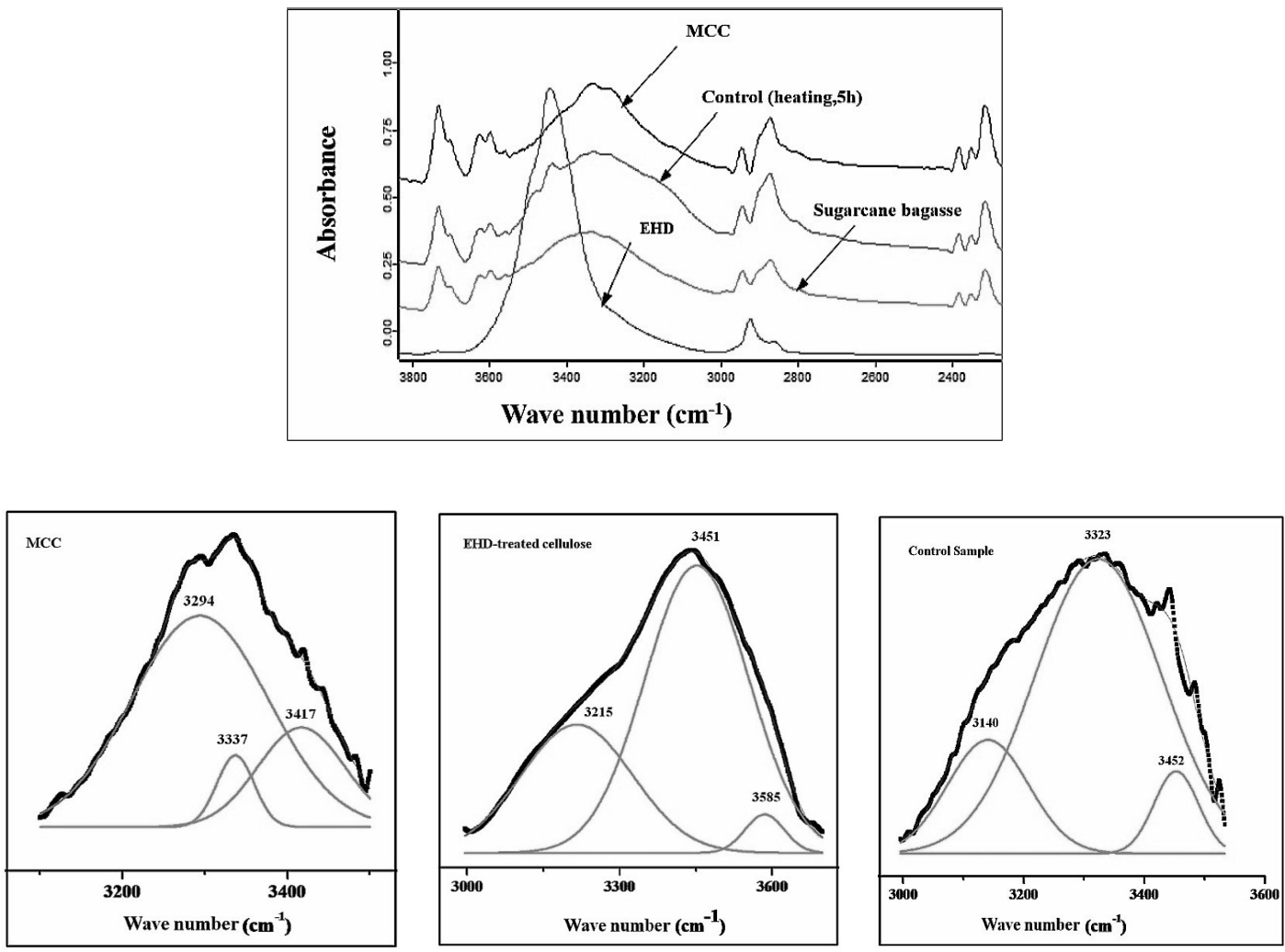

Figure 5: Deconvoluted FTIR spectra of H-bonded OH stretching region of MCC, Control and EHDtreated samples 
Therefore, it can be explained that, under the extraction conditions, the intermolecular hydrogen bonds were partially broken down at the $\mathrm{C}_{3}$ and $\mathrm{C}_{6}$ positions, which resulted into considerable variations in the strength of the hydrogen bonds of cellulose hydroxyl groups and the network.

\section{CONCLUSION}

EHD was used as a complementary technique to the conventional extraction process of cellulose. The extraction conditions were optimized using RSM. The regression was significant and the variation of responses was adequately explained by the obtained models. The mechanical rupture under the effect of EHD led to an increase in the yield of cellulose extraction. Moreover, a larger hydrodynamic size of cellulose was achieved under the optimum conditions, probably due to the amorphous structure of cellulose in which the amount of hydrogen bonds increased to a high level (according to FTIR analysis) and caused the size of cellulose to increase. Moreover, FTIR analysis indicated that EHD probably altered the proportion of inter- and intra-molecular hydrogen bond types significantly.

\section{REFERENCES}

1 C. F. Liu, J. L. Ren, F. Xu, J. J. Liu, J. X. Sun et al., Agric. Food Chem., 54, 5742 (2006), DOI: 10.1021/jf060929o

2 X. F. Sun, P. Fowler, M. Rajaratnam and G. Zhang, Phytochem. Anal., 21, $406 \quad$ (2010), https://doi.org/10.1002/pca.1211

3 I. Moussa, R. Khiari, A. Moussa, M. F. Mhenni and M. N. Belgacem, Cellulose Chem. Technol., 52, 841 (2018),

http://www.cellulosechemtechnol.ro/pdf/CCT9-

10(2018)/p.841-851.pdf

4 M. Knauf and M. Moniruzzaman, Int. Sugar J., 106, 147 (2004).

5 W. Chen, H. Yu, Y. Liu, Y. Hai, M. Zhang et al., Cellulose, $\quad \mathbf{1 8}, \quad 433 \quad$ (2011), https://doi.org/10.1007/s10570-011-9497-z

6 M. Jorfi and E. J. J. Foster, Appl. Polym. Sci., 132, 41719 (2015), https://doi.org/10.1002/app.41719

7 S. Supranto, A. Tawfiequrrahman and D. E. Yunanto, Journal of Engineering Science and Technology, Special Issue on SOMCHE 2014 \& RSCE 2014 Conference, 2015, January, pp. 35-46.

8 F. Zeinaly, A. Saraeian, K. Gabov and P. Fardim, Cellulose Chem. Technol., 51, 45 (2017), http://www.cellulosechemtechnol.ro/pdf/CCT12(2017)/p.45-53.pdf
9 L. V. A. Gurgel, R. P. de Freitas and L. F. Gil,

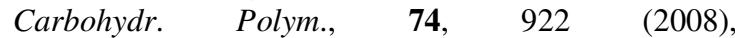
https://doi.org/10.1016/j.carbpol.2008.05.023

10 Z. M. Wang, L. Li, K. J. Xiao and J. Y. Wu, Bioresour. Technol., 100, $1687 \quad$ (2009), https://doi.org/10.1016/j.biortech.2008.09.002

11 F. Borges de Oliveira, J. Bras, M. T. Borges Pimenta, A. Aprigio da Silva Curvelo and M. N Belgacem, Ind. Crop. Prod., 93, 48 (2016), https://doi.org/10.1016/j.indcrop.2016.04.064

12 R. M. Leao, P. C. Mileo, J. M. L. L. Maia and S. M. Luz, Carbohydr. Polym., 175, 518 (2017), https://doi.org/10.1016/j.carbpol.2017.07.087

13 A. Pandey, C. R. Soccol, P. Nigam and V. T. Soccol, Bioresour. Technol., 74, 69 (2000), https://doi.org/10.1016/S0960-8524(99)00142-X

${ }_{14}$ Y. Sun and J. Cheng, Bioresour. Technol., 83, 1 (2002), https://doi.org/10.1016/S0960-8524(01)002127

15 Y. H. Ju, L. H. Huynh, N. S. Kasim, T. J. Guo, J. H. Wang et al., Carbohydr. Polym., 83, 591 (2011), https://doi.org/10.1016/j.carbpol.2010.08.022

16 A. Procentese, E. Johnson, V. Orra, A. G. Campanile, J. A. Wood et al., Bioresour. Technol., 192 , 31

(2015),

https://doi.org/10.1016/j.biortech.2015.05.053

17 M. Nuruddin, M. Hosur, M. Jamal Uddin, D. Baah and S. J. Jeelani, Appl. Polym. Sci., 133, 42990 (2016), https://doi.org/10.1002/app.42990

18 C. E. Wyman, B. E. Dale, R. T. Elander, M. Holtzapple, M. R. Ladisch et al., Bioresour. Technol., 96, 2026

(2005),

https://doi.org/10.1016/j.biortech.2005.01.018

19 Y. Asakawa, Nature, 261, 220 (1976), doi:10.1038/261220a0

${ }^{20}$ F. X. Hart and C. H. Bachman, Int. J. Biometeorol., 12, 251 (1968), https://doi.org/10.1007/BF01553425

21 Y. Chen, N. N. Barthakur and N. P. J. Arnold, Food Eng., 23, 107 (1994).

22 A. Singh, V. Orsat and V. Raghavan, Drying Technol., $\quad 30, \quad 1812 \quad$ (2012), https://doi.org/10.1080/07373937.2012.708912

23 A. Mandal and D. Chakrabarty, Carbohyd. Polym., 86, 1291 (2011),

https://doi.org/10.1016/j.carbpol.2011.06.030

24 L. N. Ludueña, A. Vecchio, P. M. Stefani and V. A. Alvarez, Fiber Polym., 14, 1118 (2013), https://doi.org/10.1007/s12221-013-1118-z

25 A. Sluiter, R. Ruiz, C. Scarlata, J. Sluiter and D. Templeton, Laboratory Analytical Procedure (LAP): Determination of Extractives in Biomass. National Renewable Energy Laboratory, Report No.: NREL/TP510-42619, 2008

26 A. Sluiter, B. Hames, R. Ruiz, C. Scarlata, J. Sluiter et al., Laboratory Analytical Procedure (LAP): Determination of Structural Carbohydrates and Lignin in Biomass. National Renewable Energy Laboratory, Technical Report NREL/TP-510-42618, 2010. 


\section{MARYAM BEYGY HARCHEGANI et al.}

27 R. Stol, J. L. Pedersoli, H. Poppe and W. T. Kok, Anal. Chem., 74, $2314 \quad$ (2002), https://doi.org/10.1021/ac0111309

${ }_{28}$ V. Webber, S. M. de Carvalho, P. J. Ogliari, L. Hayashi and P. L. M. Barreto, Ciênc. Tecnol. Aliment., 32, 812 (2012).

29 F. Peng, J. L. Ren, F. Xu, J. Bian, P. Peng et al., Food Res. Int., 43, $683 \quad$ (2010), https://doi.org/10.1016/j.foodres.2009.10.020

30 M. Nuruddin, A. Chowdhury, S. A. Haque, M. Rahman, S. F. Farhad et al., Cellulose Chem. Technol., 45, 347

(2011), http://www.cellulosechemtechnol.ro/pdf/CCT45,56(2011)/p.347-354.pdf

31 Z. He, Y. Ni and E. Zhang, Appita J., 58, 72 (2005).

32 T. Hatakeyama, Y. Izuta, S. Hirose and $H$. Hatakeyama, Polymer, 43, $1177 \quad$ (2002), https://doi.org/10.1016/S0032-3861(01)00714-5

33 M. Gáspár, G. Kálmán and K. Réczey, Process Biochem., $\quad 42, \quad 1135 \quad$ (2007), https://doi.org/10.1016/j.procbio.2007.04.003

34 M. N. M. Ibrahim, A. M. Ghani, N. Zakaria, S. Shuib and C. S. Sipaut, Macromol. Symp., 274, 37 (2008), https://doi.org/10.1002/masy.200851406

35 A. Kaushik and M. Singh, Carbohyd. Res., 346, 76 (2011), https://doi.org/10.1016/j.carres.2010.10.020

36 P. Penjumras, R. Abdul Rahman, R. A. Talib and K. Abdan, Sci. World J., Article ID 293609, (2015) DOI: $10.1155 / 2015 / 293609$.
37 J. T. Oberlerchner, T. Rosenau and A. Potthast, Molecules, $\quad 20, \quad 10313$ https://doi.org/10.3390/molecules200610313

38 Malvern Instruments Limited, Characterization of IgG monomers and their aggregates, https://www.malvernpanalytical.com/en/learn/knowled ge-center/application-notes/AN150129-IggMonomers-Aggreg.

39 M. C. Popescu, C. M. Popescu, G. Lisa and Y. Sakata, J. Mol. Struct., 988, 65 (2011), https://doi.org/10.1016/j.molstruc.2010.12.004

40 F. Carrilo, X. Colom, J. J. Suñol and J. Saurina, Eur. Polym. J., 40, $2229 \quad$ (2004), https://doi.org/10.1016/j.eurpolymj.2004.05.003

41 S. Ahmadzadeh, S. Desobry, J. Keramat and A. Nasirpour, Carbohyd. Polym., 141, 211 (2016), https://doi.org/10.1016/j.carbpol.2016.01.017

42 S. Ahmadzadeh, A. Nasirpour, M. Beygy Harchegani, N. Hamdami and J. Keramat, Carbohyd. Polym., $\quad \mathbf{1 8 8}, \quad 188 \quad$ (2018), https://doi.org/10.1016/j.carbpol.2018.01.109

43 T. Kondo and C. Sawatari, Polymer, 31, 393 (1996), https://doi.org/10.1016/0032-3861(96)82908-9 44 P. Langan, Y. Nishiyama and H. J. Chanzy, J. Am. Chem. Soc., 121, 9940 (1999), https://doi.org/10.1021/ja9916254 